\title{
A Personalized Rehabilitation System based on Wireless Motion Capture Sensors
}

\author{
Pedro Macedo ${ }^{1,2}$, José A. Afonso ${ }^{1}$ and Ricardo Simoes ${ }^{2,3,4}$ \\ ${ }^{1}$ Department of Industrial Electronics, University of Minho, Guimarães, Portugal \\ ${ }^{2}$ Institute for Polymers and Composites IPC/I3N, University of Minho, Guimarães, Portugal \\ ${ }^{3}$ Life and Health Sciences Research Institute (ICVS/3Bs), University of Minho, Braga, Portugal \\ ${ }^{4}$ School of Technology, Polytechnic Institute of Cávado and Ave, Barcelos, Portugal \\ \{pmacedo,jose.afonso\}@dei.uminho.pt,rsimoes@dep.uminho.pt,rsimoes@ipca.pt
}

Keywords: $\quad$ Motion Capture, Physiotherapy, Wireless Sensor Networks, Wearable Sensors.

\begin{abstract}
We live in an aging society, an issue that will be exacerbated in the coming decades, due to low birth rates and increasing life expectancy. With the decline in physical and cognitive functions with age, it is of the utmost importance to maintain regular physical activity, in order to preserve an individual's mobility, motor capabilities and coordination. Within this context, this paper describes the development of a wireless sensor network and its application in a human motion capture system based on wearable inertial and magnetic sensors. The goal is to enable, through continuous real-time monitoring, the creation of a personalized home-based rehabilitation system for the elderly population and/or injured people. Within this system, the user can benefit from an assisted mode, in which their movements can be compared to a reference motion model of the same movements, resulting in visual feedback alerts given by the application. This motion model can be created previously, in a 'learning phase', under supervision of a caregiver.
\end{abstract}

\section{INTRODUCTION}

Physiotherapy is a therapy which aims to help recover movement and restore normal body function, when a person is affected by illness, injury or disability. Musculoskeletal, neuromuscular, cardiovascular and respiratory are some of the physical problems physiotherapists can help treat.

Both physical and cognitive functions decline with aging. Compensatory and preventive management can control the effects of physiological system decline. Generally people reach their peak of performance abilities and health at ages between adolescence and 30 years. After this period, it is known that functional capacity declines throughout the person's lifespan, depending on genetics, lifestyle and overall health (Nitz and Hourigan, 2004).

Physiotherapy plays an important role in this aging society. Well trained health care professionals in this area can assist the patient in the rehabilitation process and improve his quality of life. It is important to maintain constant physical activity, in order to preserve movement ability, physical adaptability, mobility and coordination of the individual, by his own means or with assistance, whether at home or in an assisted living facility. Movements can be categorized within three levels: active movement, which describes the movement that a can be initiated and controlled independently; assisted active, where in order to achieve a complete movement some initial and control movement effort has to be taken from the resident, with the caregiver assistance; and passive movement, where all the movement is performed by the caregiver. Among the many benefits of doing exercise, we can cite the improvement of strength, increased range of motion, improved flexibility, improved functional mobility, increased self-esteem, improved posture, improved gait, improved efficiency of movement and improved quality of life (Nitz and Hourigan, 2004).

Taking this into consideration, this project aims to create a platform which enables and supports the practice of physical activity; and/or serve as a support platform for home-based physical rehabilitation, providing feedbacks concerning the correctness of the executed movement.

In the case of rehabilitation, the physiatrist and/or the physiotherapist will assess the nature of the patient's problems, set goals based on the extent 
of those problems, provide an adequate treatment and continuously evaluate the patient's progress. Physiatry, or rehabilitation medicine, has as one of its main aspects the recognition of functional deficits caused by injury or illness. The identification of these deficits is of utmost importance, in order to best implement a proper treatment program to restore the patient's performance (Braddom, 2006). A superior treatment can only be taken if the physiatrist is fully aware of the patient's history and difficulties he may have conducting the prescribed exercises. According to the author (Braddom, 2006), there are seven levels of disabilities. The level 1 of the scale is the most critical case, where total assistance is needed and the patient expends less than $25 \%$ of the effort. The scale from 1 to 5 encompasses a dependent disability, meaning that the patient requires another person to either supervise or assist in the performed activity; whereas in scales 6 to 7 assistance in not necessary to perform the activity.

Normal activities of daily living (ADL) like bathing and showering, dressing, eating and functional mobility, may suffer due to any existing physical problem. The combination of these problems, adding to the effort that some activities, like standing up, sitting down or picking up things, exert in our body joints, leads, in extreme cases, to the use of a prosthetic device to preserve the person's quality of life. Total hip replacement and rehabilitation is a perfect example of the interaction that should exist between the patient and his caregiver. After the surgery, in order to achieve a satisfactory level of functionality and independence in the patient's daily life, it is important to continue with the prescribed daily exercises. Patients with hip replacement have limited motion amplitude, so movements have to be restricted during the first three months after the surgery. Flexion above $90^{\circ}$ and flexion combined with abduction movements should be avoided, to prevent the prosthesis displacement (Brander and Stulberg, 2006) (O'Leary et al., 2011).

The continuous growth of the ratio of elderly population compared to the total population in developed societies causes a real and possible problematic demographic change (Linz and Stula, 2012) (Stula, 2012). In order to prevent this structural demographic change, new services and products or ambient assisted living (AAL) must be created. The goal of such products is to provide better life conditions for the older generation in their environment, by increasing their self-confidence, autonomy and mobility (Sun et al., 2009)
(Fuchsberger, 2008) (Kleinberger et al., 2007).

This paper aims to present the current status of a wireless posture monitoring system, based on wearable inertial and magnetic sensors, applied to rehabilitation. The purpose is to create a personalized home-based exercise assisted mode for the elderly people. The captured movements are evaluated and compared to a prescribed exercise, with the purpose of assist the user to correctly perform the proposed movements.

This paper is organized as follows. The next section presents the related work. An overview of the implemented system is presented in Section 3. Section 4 introduces and explains the methods used to create a rehabilitation system, provided with visual feedback to the user, while section 5 presents the conclusions and discusses future work.

\section{RELATED WORK}

The authors in (Cavallo et al., 2009) describe the implementation of a pervasive intelligent system for rehabilitation, composed of a ZigBee network, with a coordinator and actuator nodes able to identify and control the patient's activities, and send warnings to a caregiver.

Within the same context, with the goal to deliver health care services to the community, a rehabilitation service operating at a person's home over a telecommunications network was developed in (Hamel et al., 2008). According to the authors, most types of telerehabilitation services fall into two categories: clinical assessment of the patient's functional abilities in his environment; and clinical therapy. The developed system was based in cameras, local and remote computers, with dedicated (and user-friendly) modular software interfaces for videoconference connections, and sensors (accelerometer and gyroscope).

The authors in ( $\mathrm{Lu}$ et al., 2013) developed a system to enable a set of balance rehabilitation exercises for patients with spinocerebellar ataxia (SCA), for continuous and safe practice at home, through information and communication technology. The system integrates physiological monitoring and feedback coaching with a telecare center that enables real-time one-to-multiple personal exercises monitored by care managers.

Unlike the last two studies, which are based on the combination of data from several cameras, to obtain a 3D location of the patient's body and limbs, this paper aims to represent a 3D model of a patient's body segments and the development of a 
system based on a wireless sensor network (WSN) and wearable inertial and magnetic sensors. When compared to a camera approach, this method has the advantage of higher flexibility and mobility, as it can be used in uncontrolled environments, without lighting or line-of-sight concerns (Aminian and Najafi, 2004).

Physiotherapy can take advantage of the monitoring of human body movements (body kinematics) in areas such as health care (to treat patients) or sports (to support the athlete recovery or improve his performance). However when creating a WSN to monitor human body motion, several factors must be taken into account in order to assure a reliable system. These factors include: energy efficiency, since normally the sensor nodes are energy constrained; sensor node fixation in the body; high amount of data generated per sensor node (unlike typical WSNs); and the impact of the human body on the wireless signal propagation. Some of the technical challenges faced at the creation of a WSN for rehabilitation purposes are described by the author in (Hadjidj et al., 2012).

The performance of inertial, magnetic and gyroscope sensors, when applied to body kinematics measurement, was underlined by several authors. A study that compares the anatomical joint angles obtained by an inertial measurement unit (IMU) to those calculated from position data of an optical tracking device is presented in (Bergmann et al., 2009). The two measurement methods were evaluated by calculating the root mean square error (RMSE) and by calculating a two-tailed Pearson product-moment correlation coefficient between the two signals. Studies show a strong correlations, range 0.93 to 0.99 , between the two signals, as well an average RMSE of 4 degrees over the joint angle. It is concluded that IMUs offers a good alternative system for measuring anatomical joint angles, by providing an opportunity to perform accurate measurements in complex real-life environments without using constrained measurement device (markers system). In (Lin and Kulic, 2012), the authors developed a kinematics system to estimate the human leg posture and velocity. The posture was captured through wearable sensors (accelerometer and gyroscope) during the performance of typical physiotherapy and training exercises. An extended Kalman filter is applied to estimate joint angles during an arbitrary three dimensional motion. This type of system enables applications such as monitoring during knee and hip rehabilitation.

The two last mentioned studies can merely serve as an alternative tool for the traditional gait analysis system based on high-speed cameras. On the other hand the system presented here, which also serves as a gait analysis, offers in addition the ability to ascertain the proper body posture and good movement execution by the user in real-time.

\section{SYSTEM OVERVIEW}

The developed wireless posture monitoring comprises multiple sensor nodes, each one attached to a monitored body segment, and a base station that sends the collected data to a personal computer (PC). The collected information consists in inertial and magnetic readings. The processing of the information in the PC enables the calculation, in real-time, of the $3 \mathrm{D}$ orientation of the module, expressed by the pitch, roll and yaw angles. Figure 1 represents the structure of the developed system.

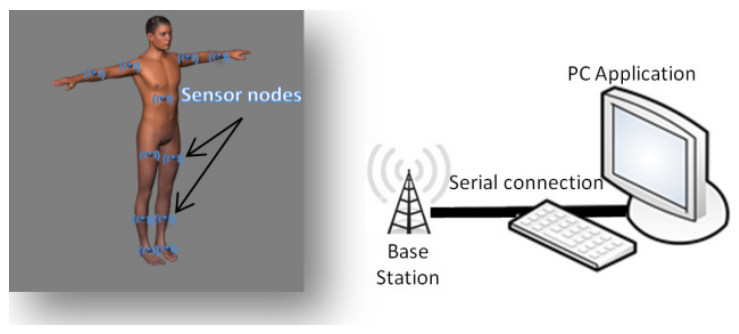

Figure 1: System components.

In this system, the CC2530, from Texas Instruments (Texas Instruments, 2009), allows the wireless communication between the base station and the sensor nodes. The CC2530 is a true systemon-chip (SoC) solution for IEEE 802.15.4 applications (IEEE Std 802.15.4, 2006) which integrates an 8051 based microcontroller and an 802.15.4 transceiver working in the license-free $2.4 \mathrm{GHz}$ frequency band.

The control of the communication through the wireless medium is achieved through the Enhanced Low Power Real Time (eLPRT) MAC protocol (Afonso et al., 2011), which was designed to optimize the quality of service (QoS) provisioning and the bandwidth utilization efficiency.

\subsection{Node Architecture}

The base station and sensors nodes have the same architectural principle, as illustrated in Figure 2, but differentiating on the communication between the microcontroller and the sensors, which is absent on the base station. 


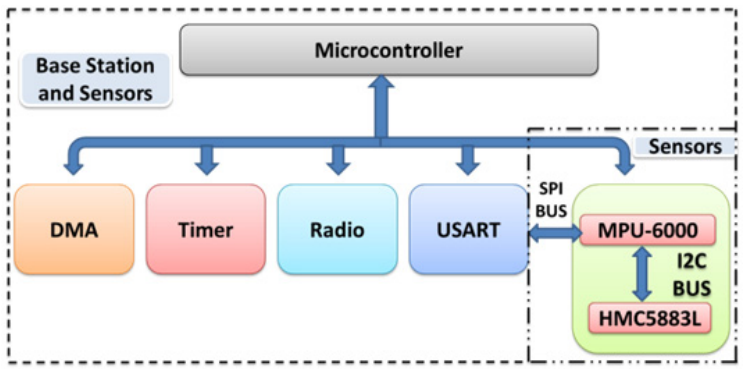

Figure 2: Base station and sensor architecture.

The Universal Synchronous Asynchronous Receiver Transmitter (USART) and Radio peripherals are controlled by the CC2530 with the assistance of the Direct Memory Access (DMA) subsystem. Some of the station base responsibilities are the association of new sensor nodes into the network, the allocation of time slots on the eLPRT superframe for the nodes transmission, and the network synchronization with the aid of periodic beacons.

The information regarding the number of readings of the sensors per superframe and the transmission period is set by the user's application. In the sensor nodes, data messages containing the readings of the 3-axis inertial, magnetic and gyroscope sensors, temperature and battery level are aggregated into a single message, according to the number of readings per superframe. The sensor information is collected through the serial peripheral interface (SPI) bus and sent to the base station once per superframe using the eLPRT protocol.

\subsection{Sensor Node Prototype}

Within the sensor prototype (Macedo et al., 2014), powered by a $3.9 \mathrm{~V} / 120 \mathrm{mAH}$, two sensors can be found, the MPU-6000 Motion Processing Unit from InvenSense, which has an embedded 3-axis MEMS accelerometer, a 3-axis gyroscope (readings not used at the moment) and a digital motion processor (DMP) hardware accelerator engine with an auxiliary $\mathrm{I}^{2} \mathrm{C}$ port that interfaces to a third party digital sensor, such as a magnetometer. The Honeywell 3-axis Digital Compass IC HMC5883L is used in this prototype. More detailed information about the sensors is described in (Macedo et al., 2014).

\subsection{PC Software}

The system component with the greatest relevance to the user is the PC application. This application serves as a mediator between the users and the base station; it can send commands and receive information sent by the sensor nodes attached to the body segments. Upon reception of the data from the sensors, it calculates the angles of rotation and presents the movement of the user's body in realtime on a 3D model of the human body.

One sensor node per monitored segment is necessary to measure the movement, with three degrees of freedom (DOF), expressed by the Euler angles pitch, roll and yaw. The overall system was designed to be as intuitive and easy to handle by the user as possible. When compared to the previously presented application (Macedo et al., 2014), this one presents new features. The software provides means to create learning files, to later compare movements; and rehabilitation sessions files, where user information and motion angles are stored. It also introduces an assisted mode, where feedback regarding the correctness of the movements is given to the user. These session files can later be employed to evaluate the user's progress. A toolbar with the most used buttons was also included, as well as LEDs indicating that a learning session or rehabilitation is being recorded. Figure 3 presents the main window of the developed software.

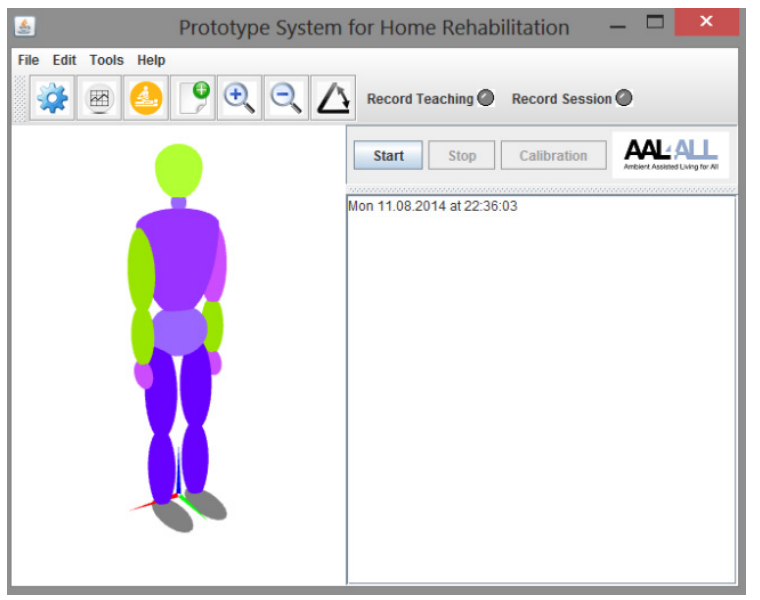

Figure 3: Application interface.

\section{SYSTEM EVALUATION}

Advances have been made regarding the application described in (Macedo et al., 2014). At this point, the developed real-time 3D Java application, not only evaluates the motion capture capabilities of the system, but can also provide movement feedback. The goal is to develop a home-based rehabilitation system that, through constant monitoring of the movement, is capable of interacting in real-time with 
the user. The purpose of this interaction is to assist the user to perform the prescribed exercises correctly. Poorly executed exercises can delay the rehabilitation process, or even cause more damage.

Thus, the created application can serve as a personalized exercise reference to a prescribed and firstly assisted session, or even generic movement body amplitudes; or merely serve to assess the patient's progress through the registration of rehabilitation log sessions (if desired, both can be performed simultaneously). This personalized exercise reference system has three stages. The first stage consists of a learning mode (section 4.1), where it is showed to the patient how to perform the exercise according to its specifications (special needs he may have). This stage can be replaced for a generic amplitude movement (correct patient positioning and plane of motion for the moved body joints), as the ones seen in (Braddom, 2006). Amplitude movements, such as shoulder flexion and extension, shoulder abduction, shoulder internal and external rotation, elbow flexion and more. The second stage (section 4.2) is characterized by the personification of each patient movement into an individual model. Finally, in the third stage (section 4.3), the patient can perform the prescribed exercises at home, through the guidance of the assisted mode.

The learning mode and personalized model will be further discussed in the sections 4.1 and 4.2 respectively. Section 4.3 will focus on the assisted mode and a more detailed explanation of how this assisted mode is achieved is described in section 4.3.1. Finally section 4.4 deals with the rehabilitation sessions.

\subsection{Learning Mode}

Within this phase the patient will be taught, by medical specialist, the correct movement that should be replicated at home during the assisted mode. This method aims to create a text file, which will serve, later on, to learn and create a motion model of a correctly performed exercise.

Each type of performed exercise creates a unique motion model, depending on the type of exercise and subject. Hence, every recorded movement is saved on a text file, containing the angles between each module present in the network and the planes of motion of the global axis system and/or the reference sensor node.

Within the learning file, a header (common to all files) is created containing the learning session number, transmission period (superframe duration), number of samples per period, date of session and a record of the angles saved to the file (the angle name given below depend on the specific movement, in this case arm):

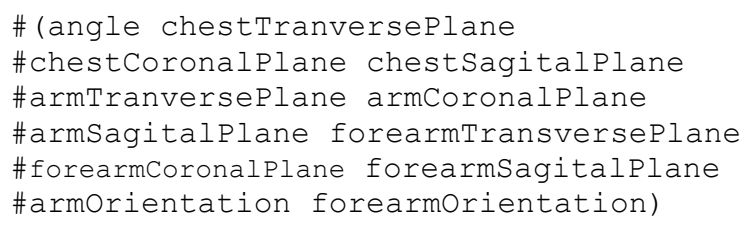

The angle mentioned in the header file it is the angle made between two segments of the body. Taking as an example the arm, the angle made between the arm and the forearm. Chest angles with the motion plane are set with the global axis system; arm and forearm angles are set with chest as reference. armorientation and forearmorientation are direction angles, calculated with the chest reference. Further along (Section 4.3.1), will explained with more detail how these angles are calculated. Later these angles will serve as a means to create the motion model to an exercise. The process of creating a new learning file is initiated by the user and/or the physiotherapist. Also, this process is protected by a password in order to prevent recording false movements. Every new reading (correspondent angle calculation) from the sensors represents a new line in the text file.

\subsection{Personalized Motion Model}

The goal of this phase is to obtain, for the individual subject and for each exercise, a personalized model of one motion cycle, which will serve as reference to the assisted exercise phase (section 4.3). After opening the file, a motion model is immediately created. This motion model is not more than the processing of the angles recorded into a text file during the learning phase.

At this moment the application only deals with uniform movements, that is, simple movements, as the ones seen in (Braddom, 2006) like: shoulder flexion and extension; shoulder abduction; elbow flexion; hip flexion, knee flexion; hip abduction; hip flexion, knee extension; knee flexion; hip internal and external rotation. The current motion model uses a global threshold system of maximum and minimum limits that an angle can take with the motion planes (global axis system and reference axis system).

\subsection{Assisted Mode}

The replication of movement through the 3D model of the human body is inherent to the application. It is 
as simple as attaching a sensor node in the body segment which you want to reproduce the movement. On the other hand, the creation of logs of rehabilitation sessions and learning processes must be initiated by the users. As opposed to these processes, the assisted mode depends on the preexistence of a learning file, so that a motion model can be created. This assisted mode consists in comparing the performed motion to a stored reference motion model of the same movement (learning file). The result of this comparison is translated to a visual feedback to the user. To start the assisted mode, the user must first open the correspondent learning file. After opening the file and correspondent motion model creation, the assisted mode and visual feedback can begin.

At home, when using the assisted mode, the application should be able to inform the user whether the movement performed previously with the caregiver, is being well executed. Figure 4 demonstrates the capabilities of the human 3D model and application to provide feedback to the user about the correctness of the movement. The yellow and red colors are used to inform the user that the allowed limits for the motion were exceeded (yellow for the first threshold and red for the second threshold). A detailed view of the angles that are exceeding their thresholds is shown in the bottom right side of the window, which presents the values of the angles that the segment makes with each motion plane. For this test, a learning file was created, a simple flexion and extension of the right arm, and then the user was asked to replicate the previously taught movement.

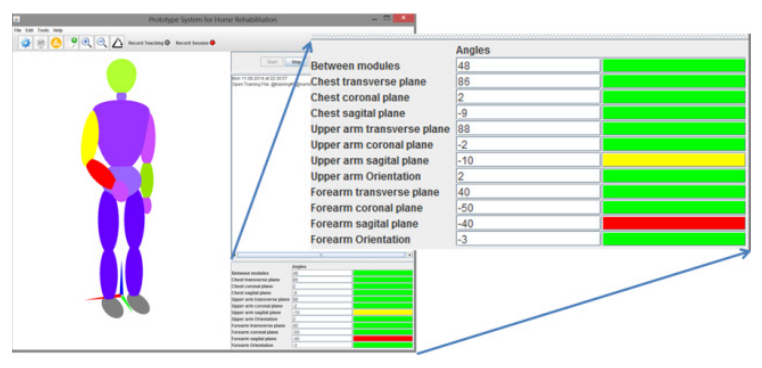

Figure 4: Application interface in assisted mode.

\subsubsection{Assessment of Range of Motion}

In (Braddom, 2006), the author provides guidelines to assess the efficacy of treatment procedures, to determine the biomechanical cause of impairment and to document the integrity of a joint range of motion. According to the author, normal range of motion (ROM) varies based on age, obesity, gender, conditioning and genetics. Moreover, men have a more limited range when compared to women, depending on age and specific joint action. In order to better assess the motion, passive ROM tests should be performed by the examiner, thorough all planes of motion, in a relaxed patient. On the other hand, active ROM tests through all planes of motion, performed by the patient without assistance from the examiner, simultaneously evaluate muscle strength, coordination of movement and functional ability.

The range of motion is measured with a universal goniometer and should be performed prior to strength testing. The developed system depicted here can serve as a test goniometer to assess patient range of motion and correctness of movement. The planes of motion mentioned earlier consist in a division of the human body into three cardinal planes, as shown in Figure 5. The sagittal plane divides the body into left and right halves. The frontal (coronal) plane divides into anterior and posterior halves. Lastly, the transverse plane divides the body into superior and inferior parts.

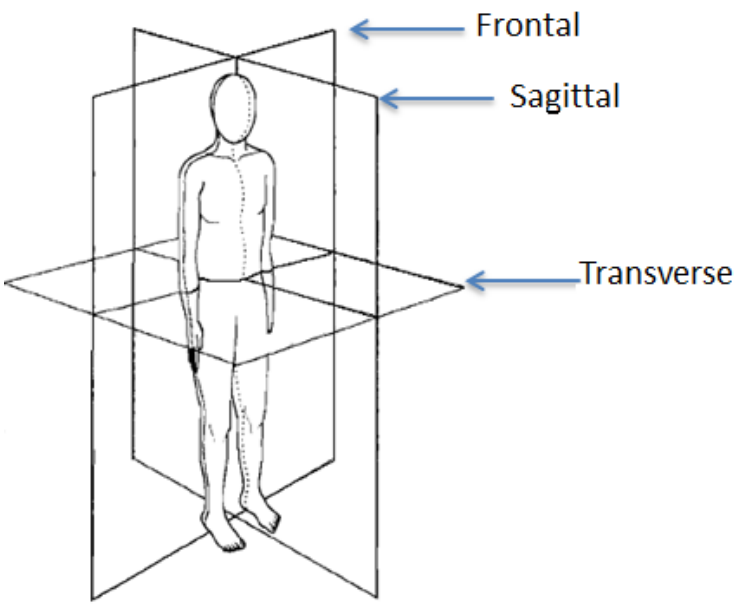

Figure 5: Cardinal planes of motion (adapted from Braddom, 2006).

The planes of motion are defined according the coordinate system as follows: the transverse plane is delimited by the $\mathrm{x}$ and $\mathrm{y}$ axis; the sagittal plane, by the $\mathrm{y}$ and $\mathrm{z}$ axis; and the coronal is bounded by the $\mathrm{x}$ and $\mathrm{z}$ axis. Using this global coordinate system, or planes, defined by the axis position of a reference module for other at the time of the movement, it is possible to calculate the range of motion along the planes. As an example the chest module can serve as a reference for the arm modules. The coordinate system used to calculate the three orientation angles was the right-handed. The forward orientation of the module is set to be along the y axis, with pitch defining the rotation on the $\mathrm{x}$ axis $\left(R_{x}(\propto)\right)$, the roll 
on the $\mathrm{y}$ axis $\left(R_{y}(\beta)\right)$ and yaw on the $\mathrm{z}$ axis $\left(R_{z}(\gamma)\right)$, according to equations 1,2 and 3 respectively. After the matrix multiplications (rotations), the final matrix of the module's orientation is found in equation 4. This matrix is needed in case the reference for the motion planes is another sensor node; within this matrix the module's axis can be found. In those cases where the reference is the global system, the axes are set to its origin: $\mathrm{x}$ axis to $\left[\begin{array}{lll}1 & 0 & 0\end{array}\right] ; \mathrm{y}$ axis to $\left[\begin{array}{lll}0 & 1 & 0\end{array}\right]$; and $\mathrm{z}$ axis to $\left[\begin{array}{lll}0 & 0 & 1\end{array}\right]$, equal to the identity matrix.

The module's orientation vector is given by multiplying the final rotation matrix by its original position $\left[\begin{array}{lll}0 & 0 & 1\end{array}\right], \mathrm{x}$ axis, $\mathrm{y}$ axis and $\mathrm{z}$ axis respectively.

$$
\begin{aligned}
R_{x}(\propto) & =\left[\begin{array}{ccc}
1 & 0 & 0 \\
0 & \cos \propto & -\sin \alpha \\
0 & \sin \propto & \cos \alpha
\end{array}\right] \\
R_{y}(\beta) & =\left[\begin{array}{ccc}
\cos \beta & 0 & \sin \beta \\
0 & 1 & 0 \\
-\sin \beta & 0 & \cos \beta
\end{array}\right] \\
R_{z}(\gamma) & =\left[\begin{array}{ccc}
\cos \gamma & -\sin \gamma & 0 \\
\sin \gamma & \cos \gamma & 0 \\
0 & 0 & 1
\end{array}\right] \\
R_{(\beta, \alpha, \gamma)} & =R_{y}(\beta) R_{x}(\alpha) R_{z}(\gamma)
\end{aligned}
$$

The orientation vector for the reference node (sensor node or not) is given by the perpendicular vector (normal vector) of the plane we want to calculate. In other words, if we want to calculate the angle between one module orientation vector and the transversal plane, the orientation vector of that plane is its $\mathrm{z}$ axis. The angle $(\varphi)$ between the two orientation vectors is calculated using equation 5 . Therefore, the mentioned angle defines the angle between a given module orientation vector and a plane of motion (Dunn et al., 2011).

$$
\varphi=\arcsin \left(\frac{\vec{V}_{\text {ref }} \cdot \vec{V}_{\text {forearm }}}{\left\|V_{\text {ref }}\right\|\left\|V_{\text {forearm }}\right\|}\right)
$$

So far, the three planes of motion gave us three angles to each module in relation to its reference(s), but a fourth angle can be calculated, the torsion angle. This angle reflects the difference between the one module and its reference(s) direction. As an example, if both modules are presented northwards, the angle between them is zero. This angle must be calculated with another module as reference, because otherwise there would be no way to predict the initial state (or direction) of the patient. The plane used in the reference module is the sagittal, and on the side of the other module it is used its direction, $\mathrm{y}$ axis. The angle is calculated using equation 5 , with the y axis being considered the orientation vector of the module; this value was obtained in the final rotation matrix of the module. The purpose of calculating all of these possible angles, for each module, relative to the motion planes, is to better assess the user's performance.

\subsection{Rehabilitation Session}

This rehabilitation session mode was created in the application in order to create session logs that can later serve to gauge the patient's progress during the rehabilitation process. A new rehabilitation session must be initiated by the user; only this way the application will automatically create the new session file.

Several experiments were carried out to evaluate the developed system. The one presented in this paper had three sensor nodes attached to the body, two of them on the right arm (upper arm and forearm) and the other on the chest. The goal was to verify the replication of the body movement in the 3D model present in the application and, at the same time, produce a rehabilitation session file. Figure 6 and Figure 7 exemplify these experiments by means of photographs taken while the movement was being executed.

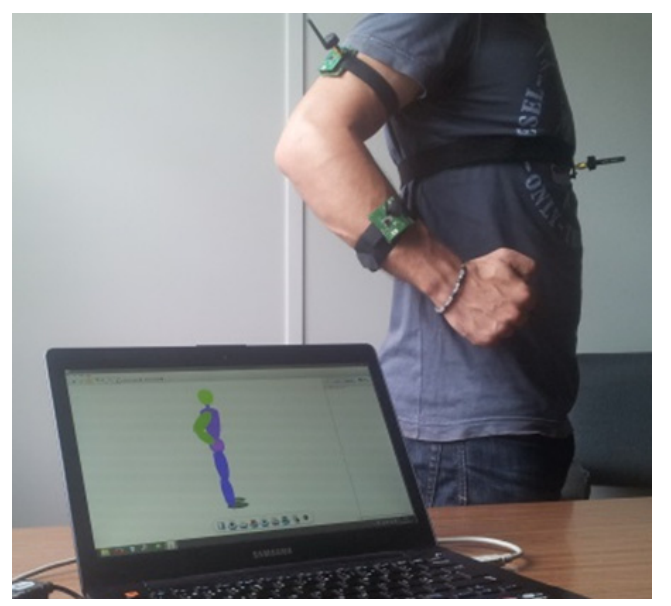

Figure 6: Right arm movement replication, first position.

Figure 8 presents the angle between the upper and forearm along the time. The user was requested to do a simple movement, flex and extend the arm, multiple times. Information regarding the user name, session number, type of rehabilitation, superframe period, number of samples per superframe and date of session can be seen on the left side. In the future, more body angles will be added to the chart, according to the performed movement and including 
only the most relevant angles from those recorded on the text file.

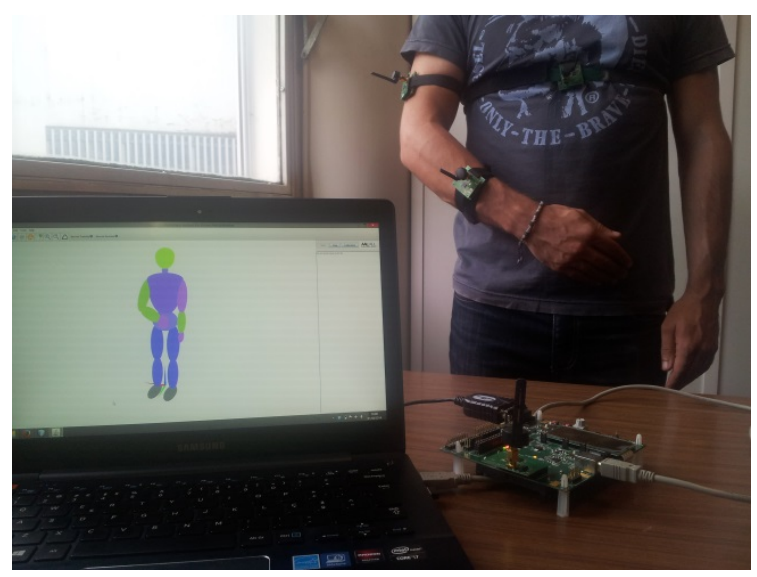

Figure 7: Right arm movement replication, second position.

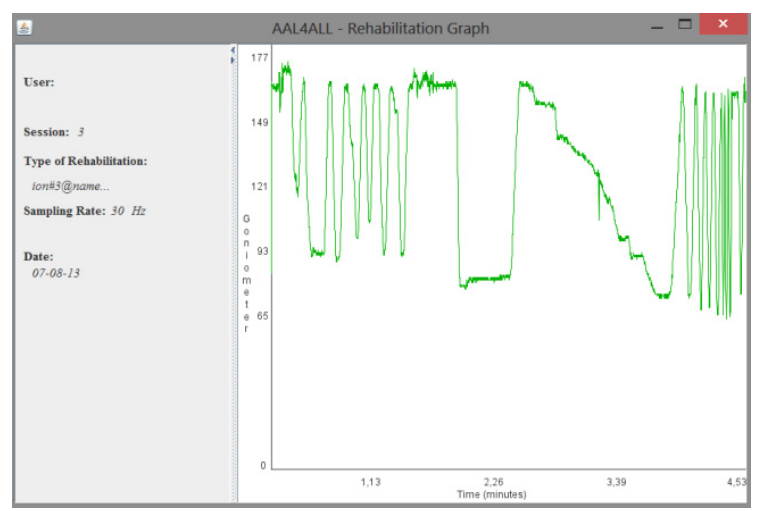

Figure 8: Chart of the angle of the articulation.

\section{CONCLUSIONS}

This paper outlines the design and development of a wireless sensor network based on wearable and easily placed inertial and magnetic sensors, which is applied to monitoring of physical activities in clinical settings and home environments. Such a system can have tremendous application to complement and enhance physical rehabilitation procedures. An application capable of collecting data from the sensors and present them in a real-time 3D model of a human body is under development, focusing on the special case of assisting elderlies in the practice of physiotherapy at home.

The main objective of this project consists in developing a home-based rehabilitation system that, through constant monitoring of the movement, is capable of interacting in real-time with the user. The purpose of this interaction is to assist the user to perform the prescribed exercise correctly. The rehabilitation process can be delayed or even cause more damage if the exercise is poorly performed. Therefore, this system can help medical specialists to take into account physical limitations of the patients in the preparation of physiotherapy sessions, and to set well-defined objectives with regard to patient outcomes.

The caregiver presence is not required during the home-based sessions (assisted mode), but only in the first session (learning mode). Unlike camera-based systems, the patient can freely move anywhere under the coverage of the WSN.

As future work, it is intended to create graph sessions providing more useful information, such as other meaningful angles, in order to create rehabilitation session files that can better serve the caregiver when assessing the patient's progress.

Another feature under study is the compensation of misplacement of the modules in the human body, that is, if the sensor nodes are not attached correctly to the chosen body segment, through the automatic detection and correction of angle offsets with relation to the correct position.

Studies are also being carried out in order to improve the existing motion model. So far, a global thresholds technique is being used, which places limits to the angles which are used throughout the movement and provides feedback to the user about the correctness of his motion. Although this solution results for simple movements, it is not able to account for more complex movements. Therefore, a new motion model based on local thresholds and state machines is currently being implemented.

\section{ACKNOWLEDGEMENTS}

Project "AAL4ALL", co-financed by the European Community Fund FEDER through COMPETE Programa Operacional Factores de Competitividade (POFC). FCT - Foundation for Science and Technology - Lisbon, Portugal, through project PEst-C/CTM/LA0025/2013.

\section{REFERENCES}

Afonso, J., Silva, H., Macedo, P., Rocha, L., 2011. An Enhanced Reservation-Based MAC Protocol for IEEE 802.15.4 Networks. Sensors, Vol. 11, Issue 4, April 2011, pp. 3852-3873. 
Aminian, K., Najafi, B., 2004. Capturing human motion using body-fixed sensors: outdoor measurement and clinical applications. In Computer Animation and Virtual Worlds, Volume 15, Issue 2, pp. 79-94.

Bergmann, J., Mayagoitia, R, Smith, I., 2009. A portable system for collecting anatomical joint angles during stair ascent: a comparison with an optical tracking device. In Dynamic Medicine 2009, 8:3.

Braddom, R. L., 2006. Physical Medicine \& Rehabilitation. Third Edition.

Brander, V., Stulberg, S. D., 2006. Rehabilitation After Hip and Knee Joint Replacement. An Experience and Evidence-Based Approach to Care. In American Journal of Physical Medicine \& Rehabilitation, Vol. 85, No. 11 (Supplement): S98-118.

Cavallo, F., Aquilano, M., Odetti, L., Arvati, M., Carrozza, M.C., 2009. A first step toward a pervasive and smart ZigBee sensor system for assistance and rehabilitation. IEEE $11^{\text {th }}$ International Conference on Rehabilitation Robotics Kyoto International Conference Center, Japan, June 23-26, 2009.

Dunn, F., Parberry, I., 2011. 3D Math Primer for Graphics and Game Development. Second Edition pp. 141-143, ISBN-13: 978-1-4398-6981-9, 2011.

Fuchsberger, M., 2008. Ambient Assisted Living: Elderly People's Needs and How to face Them. In Proceedings of the $1^{\text {st }}$ ACM international workshop on Semantic ambient media experiences.

Hadjidj, A., Souil, M., Bouadballah, A., Challal, Y., Owen, H., 2012. Wireless sensor networks for rehabilitation applications: Challenges and opportunities. In Journal of Network and Computer Applications.

Hamel, M., Fontaine, R., Boissy, P., 2008. In-Home Telerehabilitation for Geriatric Patients. IEEE Engineering in Medicine and Biology Magazine. July/August 2008.

IEEE Std 802.15.4-2006, Part 15.4: Wireless Medium Access Control (MAC) and Physical Layer (PHY) Specifications for Low-Rate Wireless Personal Area Networks (WPANs), September 2006.

Instituto Nacional de Estatística, Statistics Portugal, 2014. Resident population in Portugal with a decreasing and aging trend. Press release, 10 July 2014.

Kleinberger, T., Becker, M., Ras, E., Holzinger, A., 2007. Ambient Intelligence in Assisted Living: Enable Elderly People to Handle Future Interfaces. In $4^{\text {th }}$ International Conference on Universal Access in Huma-Computer Interaction, UAHCI.

Lin, J., Kulic, D., 2012. Human pose recovry using wireless inertial measurement units. In Physiologial Measurement, Volume 33, Number 12, 2012.

Linz, K., Stula, S., 2012. Demographic change in Europe An Overview. Working Paper No. 4 of the Observatory for Sociopolitical Developments in Europe.

Lu, T., Liu, H., Chen, R., Chen, Y., 2013. MotionSensisng Based Management System for Smart Context-Awareness Rehabilitation Healthcare. In Advances in Internet of Things, 2013, 3, 1-7.
Macedo, P., Afonso, J. A., Rocha, L. A., Simões, R., 2014. A Telerehabilitation System Based on Wireless Motion Capture Sensors. International Conference on physiological Computing Systems, 7-9 January, 2014.

Nitz, J. C., Hourigan, S. R., 2004. Physiotherapy Practice in Residential Aged Care. ISBN 075068772 X.

O'Leary, K., UW Health Sports Medicine physician group, 2011. Rehabilitation Guidelines for Surface Replacement Arthroplasty (SRA). University of Winsconsin Sports Medicine.

Stula, S., 2012. Living in Old Age in Europe - Current Developments and Challenges. Working Paper No. 7 of the Observatory for Sociopolitical Developments in Europe.

Sun, H., De Florio, V., Gui, N., Blondia, C., 2009. Promises and Challenges of Ambient Assisted Living Systems. In Information Technology: New Generations. ITNG'09.

Texas Instruments, "CC2530 data Sheet" April 2009. Retrieved from http://www.ti.com. 